\title{
Three-Dimensional Laminar Boundary Layer with Small Cross-Flow*
}

\author{
ARTUR MAGER $†$ \\ California Institute of Technology
}

\begin{abstract}
Important problems involving three-dimensional boundary layer occur in almost all internal and external aerodynamic configurations. For many of these, the flow outside the boundary layer may be resolved into a large principal component and a small crosswise velocity. In this paper, three-dimensional laminar boundary-layer flows over flat and curved surfaces are treated under such a simplification. For flat surfaces, the solutions demonstrate the effect of the free stream turning on the velocity profiles in the crosswise and primary flow directions. When the surface curvature is large and varies so as to resemble a corner, the computed examples show the manner in which the asymmetric behavior of the boundary layer results from the cross-flow. The detailed examples are chosen to illustrate flows occurring on the casing and in the blade fillets of turbomachinery.
\end{abstract}

\begin{tabular}{|c|c|}
\hline \multicolumn{2}{|c|}{ Symbols } \\
\hline$a$ & $=$ cylinder radius \\
\hline$A_{m} ; B_{n} ; E_{r}$ & $=$ functions of $z$ \\
\hline$b$ & $=$ vector position \\
\hline$c$ & $=$ curvature \\
\hline$d_{i} ; l_{n, i} ; l_{n, i}(i=0,1,2, \ldots)$ & $=$ functions of $\eta$ \\
\hline$D, H$ & $=$ constants \\
\hline $\begin{array}{l}f_{m} ; f_{n} ; f_{r} ; f_{m, e} ; g_{n} ; g_{r} \\
F\end{array}$ & $\begin{array}{l}=\text { functions of } \eta \\
=\text { Blasius function }\end{array}$ \\
\hline$G_{i}(i=1,2,3)$ & $=$ body forces \\
\hline$h_{i}(i=1,2,3)$ & $=$ length stretching factors \\
\hline$L$ & $=$ length of duct \\
\hline$m, n, r$ & $=$ exponents of $x$ \\
\hline$M$ & $=$ Mach Number \\
\hline$p$ & $=$ pressure \\
\hline$q$ & $=$ vector velocity \\
\hline $\bar{q}$ & $=$ vector velocity at $y=\delta$ \\
\hline$R$ & $=$ cylinder path radius \\
\hline$R e$ & $=$ Reynolds Number $U^{0} \xi / \nu$ \\
\hline$s$ & $=$ width of duct \\
\hline$u, v, w$ & $\begin{array}{l}=\text { velocities in the boundary } \\
\text { layer }\end{array}$ \\
\hline$U, V, W$ & $=$ velocities of external flow \\
\hline$x, y, z$ & $=$ Cartesian coordinates \\
\hline$X, Y, Z$ & $\begin{array}{l}=\text { coordinates in compressible } \\
\text { flow }\end{array}$ \\
\hline$\alpha$ & $=$ yaw angle \\
\hline$\beta$ & $\begin{array}{c}=\text { angle between tangent to } \\
\zeta \text { axis and } z \text { axis }\end{array}$ \\
\hline
\end{tabular}

Presented at the Aerodynamics Session, Twenty-Second Annual Meeting, IAS, New York, January 25-29, 1954.

* This paper is partly based on a Doctoral Thesis at the California Institute of Technology and is sponsored by the Air Research and Development Command under Contract No. AF 18(600)-178. Special acknowledgment is due to Profs. F. E. Marble and $\mathrm{H}$. S. Tsien for their valuable advice and constructive criticism.

$\dagger$ Formerly, Research Fellow in Jet Propulsion; now, Engineering Specialist, Marquardt Aircraft Co.

$\gamma$
$\delta$
$\xi, \lambda, \zeta$
$\eta$
$\theta$
$\vartheta$
$\kappa$
$\nu$
$\rho$
$\chi$
$\psi ; \varphi$
$\omega$
$\omega_{i}(i=1,2,3)$
$\Omega_{i}(i=1,2)$
$\nabla$

Superscripts

$0=$ basic flow also standard quantity

* = total value (basic + perturbation)

' $=$ differentiation with respect to $\eta$

$-=$ compressible flow

Subscripts

$\delta=$ at $y=\delta$

Coordinates indicate partial differentiation

\section{(I) InTRODUCTION}

$\mathrm{T}$ HE IMPORTANT PROBLEm of three-dimensional boundary-layer flow occurs in almost any real, external or internal, aerodynamic configuration. Because of its complexity, this problem is generally avoided, and either a two-dimensional boundary-layer flow analysis is made as an approximation, or none at all. Such procedure is usually satisfactory as long as the design is conservative and the fluid is asked to perform but moderate tasks. When, however, one attempts to increase the performance by requiring the fluid to flow against stronger pressure gradients, then the available theories completely break down, with rather undesirable results. This is particularly well illustrated by the design of turbomachinery, which is successful, provided the stages of these machines operate at extremely moderate pressure ratios; far below those obtainable in cascades and single-stage experiments. However, when high pressure ratio per stage machine is attempted, the real fluid effects become so 
important that they overshadow the aims of the newly incorporated features and the designed performance cannot be realized. Consequently, the important engineering problem of increase in power output, per unit weight of the machinery, remains unsolved.

The real fluid effects demonstrate themselves primarily in the so-called secondary flow and in the threedimensional boundary-layer flow. The term secondary flow is currently used to designate flows arising from nonuniform total pressure or enthalpy, in regions not bordering on physical boundaries, where the effect of viscosity may be neglected. Such flows usually occur in internal aerodynamic configurations. On the other hand, by the term three-dimensional boundary-layer flow, we understand similar flows in the relatively thin regions, next to solid walls, where the viscosity plays an important part. Three-dimensional boundarylayer flows occur for both the internal, as well as external, aerodynamic configurations, differing in the nature of the flow outside the boundary layer. In internal aerodynamic configurations, the flow outside the boundary layer may be secondary and thus rotational, while in the external aerodynamic configurations that flow is potential.

A critical review of the three-dimensional boundarylayer literature reveals a definite division into three separate groups. The first group consists of the investigations of the forms of the boundary-layer equations and discussion of boundary-layer behavior as inferred from these forms, without actual solution of the equations. Here significant work has been done by Howarth, ${ }^{1}$ Hayes, ${ }^{2}$ and Moore. ${ }^{3}$ The second group consists of general, but approximate, treatment of three-dimensional boundary-layer flow by the momentum-integral method. The laminar case in this group has been investigated by Timman, ${ }^{4}$ and the turbulent one by Mager. ${ }^{5}$ The validity of these methods depends to a large extent on the choice of the velocity distributions in the boundary layer, and since the experimental information is meager, attempts have been made to study the velocity distributions analytically. This leads us to the third group, which consists of solutions of some specialized cases of three-dimensional laminar boundary-layer flow. These specialized cases fall into two categories: (a) where the changes with respect to one of the independent coordinates are zero, or where there exists some sort of symmetry, and (b) where there is some characteristic parameter that is small. In the first category belongs the work of Sears, ${ }^{6}$ Bödewart, ${ }^{7}$ Howarth, ${ }^{8}$ Loos, ${ }^{9}$ and Carrier, ${ }^{10}$ while in the second one the publications of Moore ${ }^{11,12}$ and Fogarty ${ }^{13}$ may be mentioned.

The general treatment of three-dimensional boundary-layer flow is extremely difficult in view of the complexity of the equations. However, for many important problems involving three-dimensional boundary layer, the flow outside the boundary layer may be resolved into a large principal component and a small cross-wise velocity. Since the pressure variation in the direction normal to the wall is usually negligible in the boundary layer, the flow there is affected by pressures existing outside the layer-that is, those governing the external flow. Consequently, the velocities in the boundary layer will have the order of magnitude of their values in the external flow. When the crosswise velocity is small as compared with the principal velocity, but otherwise not specified, the boundary layer will be only slightly three-dimensional, and the small perturbation procedure will be applicable to a great variety of external flows. The object of this paper is to treat the three-dimensional laminar boundary-layer flows over flat and sharply curved surfaces under just such simplification.

\section{(II) Boundary-Layer Flow over Flat Surface}

\section{Boundary-Layer Approximation}

The motion of an incompressible steady viscous fluid, flowing relative to a coordinate system rotating with a uniform angular velocity is described by the momentum equations,

$$
\begin{aligned}
& -\boldsymbol{q} \times(\boldsymbol{\nabla} \times \boldsymbol{q})+\boldsymbol{\omega} \times(\boldsymbol{\omega} \times \boldsymbol{b})+2 \boldsymbol{\omega} \times \boldsymbol{q}= \\
& -\boldsymbol{\nabla}\left(\frac{1}{\rho} p+\frac{1}{2} \boldsymbol{q}^{2}\right)+\nu[\nabla(\boldsymbol{\nabla} \cdot \boldsymbol{q})-\nabla \times(\boldsymbol{\nabla} \times \boldsymbol{q})]
\end{aligned}
$$

and the equation of continuity,

$$
\boldsymbol{\nabla} \cdot \boldsymbol{q}=0
$$

Making the usual boundary-layer simplifications - that is, neglecting all terms of $0(\delta)$ as compared with those of $0(1)$, - one obtains from Eqs. (1) and (2) the boundary layer equations,

$$
\left.\begin{array}{r}
u^{*} u_{x}{ }^{*}+v^{*} u_{y}{ }^{*}+w^{*} u_{z}{ }^{*}+2 \omega_{2} w^{*}-\omega^{2} \chi \chi_{x}= \\
-\frac{1}{\rho} p_{x}+\nu u_{y y}{ }^{*} \\
2\left(\omega_{3} u^{*}-\omega_{1} w^{*}\right)-\omega^{2} \chi \chi_{y}=-\frac{1}{\rho} p_{y} \\
u^{*} w_{x}^{*}+v^{*} w_{y}^{*}+w^{*} w_{z}^{*}-2 \omega_{2} u^{*}-\omega^{2} \chi \chi_{z}= \\
-\frac{1}{\rho} p_{z}+\nu w_{y y}^{*}
\end{array}\right\}
$$

and

$$
u_{x}^{*}+v_{y}^{*}+w_{z}^{*}=0
$$

These equations differ from those used in two-dimensional theory, not only by the presence of an additional equation in the $z$ direction (for notation see Fig. 1) but also by $p_{y}$ being of $0(1)$ instead of $0(\delta)$. This occurs because a pressure gradient is necessary to balance the effect of the centripetal and Coriolis forces. But the total change of pressure throughout the boundary layer along a normal to the wall is still of $0(\delta)$, and although this is larger than on a nonrotating flat plate [where it is of $O\left(\delta^{2}\right)$, it may still be neglected. A reasonable as- 
sumption, therefore, is to consider $p$ as a function of $x$ and $z$ only.

\section{External Flow}

The external flow is defined as the flow where the effects of viscosity may be neglected, regardless of its character, which may be rotational or irrotational. This definition is different from that used in the conventional mechanics of the boundary-layer flow, and it implies a division of the total flow field into a region where the effects of viscosity are neglected and a (still thinner) region where such effects are considered. If the external flow is rotational, the Etrlerian equations must be used (instead of the conventional Bernoulli's relation) for the variation of pressure,

$$
\begin{aligned}
-\overline{\boldsymbol{q}} \times(\boldsymbol{\nabla} \times \overline{\boldsymbol{q}})+\boldsymbol{\omega} \times(\boldsymbol{\omega} \times b) & +2 \boldsymbol{\omega} \times \overline{\boldsymbol{q}}= \\
& -\boldsymbol{\nabla}\left(\frac{1}{\rho} p+\frac{1}{2} \overline{\boldsymbol{q}}^{2}\right)
\end{aligned}
$$

In the present analysis it will be assumed that the external flow may be only slightly rotational, so that the derivatives $U_{y}{ }^{*}$ and $W_{y}{ }^{*}$ are small as compared with $U_{x}{ }^{*}$. This simplification permits one to specify the pressure in terms of $U^{*}, W$, and $\omega$ alone, even if $V^{*}$ is of $0(\delta)$. The external flow components $U^{*}, W^{*}$, and the angular velocity $\omega$ are assumed known. In particular one takes:

$$
\begin{aligned}
& U^{*}=U^{0}+U(x, z) ; \quad W^{*}=W(x, z) ; \\
& \boldsymbol{\omega}=\boldsymbol{i} \omega_{1}+j \boldsymbol{\omega}_{2}+\boldsymbol{k} \boldsymbol{\omega}_{3} \\
& U \ll U^{0} ; \quad W \ll U^{0} ; \quad \omega_{2} \ll \omega_{1}, \omega_{3}
\end{aligned}
$$

as describing the external flow and the angular velocity. The velocity $U^{0}$ and the angular velocity $\omega$ are assumed constant. The component $\omega_{2}$ is taken of the same order when compared to $\omega_{1}$ as $U$ when compared with $U^{0}$.

\section{Perturbed Flow Equations}

Corresponding to Eqs. (4), the flow in the boundary layer is expressed by

$$
\left.\begin{array}{rl}
u^{*} & =u^{0}(x, y)+u(x, y, z) \\
v^{*} & =v^{0}(x, y)+v(x, y, z) \\
w^{*} & =w(x, y, z)
\end{array}\right\}
$$

where again the perturbation terms $u, v, w$ are assumed small, when compared with the basic flow terms $u^{0}$ and $v^{0}$. It is clear from the expressions (4) and (5), that, since $U^{0}$ is a constant, the substitution into Eqs. (1a) and (2a) must result in the zeroth-order terms forming the Blasius "flat-plate" equations. The first-order terms then give the relations, for the perturbation velocities $u, v, w$,

$$
\left.\begin{array}{l}
u^{0} u_{x}+u u_{x}^{0}+v^{0} u_{y}+v u_{y}{ }^{0}=U^{0} U_{x}+\nu u_{y y} \\
u^{0} w_{x}+v^{0} w_{y}+2 \omega_{2}\left(U^{0}-u^{0}\right)=U^{0} W_{x}+\nu w_{y y} \\
u_{x}+v_{y}+w_{z}=0
\end{array}\right\}
$$

The solution for the basic flow is known: $u^{0}=U^{0} F^{\prime}$, $v^{0}=(1 / 2)\left(\sqrt{\left.\nu U^{0} / x\right)}\left(\eta F^{\prime}-F\right), \eta=y \sqrt{U^{0} / \nu x}\right.$, with $F(\eta)$ being the usual Blasius function tabulated in reference 14. Furthermore, proceeding in the manner of reference 3 , the equation of continuity may be identically satisfied by introduction of two functions $\psi$ and $\varphi$

$$
u \equiv \psi_{y} ; \quad w \equiv \varphi_{y} ; \quad v \equiv-\left(\psi_{x}+\varphi_{z}\right)
$$

One now assumes that the external flow velocity components and $\omega_{2}$ are expressible as power functions of the $x$ coordinate: $U=\sum_{m} A_{m}(z) x^{m}, W=\sum_{n} B_{n}(z) x^{n}$, $\omega_{2}=\sum_{r} E_{\tau}(z) x^{r}$, and puts the functions $\varphi$ and $\psi$ in a form that will later prove convenient,

$$
\begin{aligned}
\psi=\sqrt{\frac{\nu x}{U^{0}}}\left[\sum_{m} A_{m} x^{m} f_{m}+\sum_{n} \frac{\partial B_{n}}{\partial z} x^{n+1} f_{n}+\right. \\
\left.2 \sum_{r} \frac{\partial E_{r}}{\partial z} x^{r+2} f_{r}\right] \\
\varphi=\sqrt{\frac{\nu x}{U^{0}}\left[\sum_{n} B_{n} x^{n} g_{n}+2 \sum_{r} E_{r} x^{r+1} g_{r}\right]}
\end{aligned}
$$

Here $f_{m}, f_{n}, f_{r}, g_{n}$, and $g_{r}$ are functions of $\eta$ only. Using Eqs. (8) and the expressions for the basic flow velocities, Eqs. (6) become

$$
\begin{aligned}
\left\{\sum _ { m } A _ { m } x ^ { m } \left[f_{m}{ }^{\prime \prime \prime}+\right.\right. & \left.\left.\frac{F}{2} f_{m}^{\prime \prime}-m F^{\prime} f_{m}{ }^{\prime}+\left(m+\frac{1}{2}\right) F^{\prime \prime} f_{m}+m\right]\right\}+ \\
& \left\{\sum_{n} \frac{\partial B_{n}}{\partial z} x^{n+1}\left[f_{n}^{\prime \prime \prime}+\frac{F}{2} f_{n}^{\prime \prime}-(n+1) F^{\prime} f_{n}{ }^{\prime \prime}+\left(n+\frac{3}{2}\right) F^{\prime \prime} f_{n}+F^{\prime \prime} g_{n}\right]\right\}+ \\
& \left\{2 \sum_{r} \frac{\partial E_{r}}{\partial z} x^{r+2}\left[f_{r}^{\prime \prime \prime}+\frac{F}{2} f_{r}^{\prime \prime}-(r+2) F^{\prime} f_{r}^{\prime}+\left(r+\frac{5}{2}\right) F^{\prime \prime} f_{r}+F^{\prime \prime} g_{r}\right]\right\}=0 \\
\left\{\sum _ { n } B _ { n } x ^ { n } \left[g_{n}{ }^{\prime \prime \prime}+\right.\right. & \left.\left.\frac{F}{2} g_{n}^{\prime \prime}-n F^{\prime} g_{n}^{\prime}+n\right]\right\}+\left\{2 \sum_{r} E_{r} x^{r+1}\left[g_{r}^{\prime \prime \prime}+\frac{F}{2} g_{r}^{\prime \prime}-(r+1) F^{\prime} g_{r}{ }^{\prime}-\left(1-F^{\prime}\right)\right]\right\}=0
\end{aligned}
$$




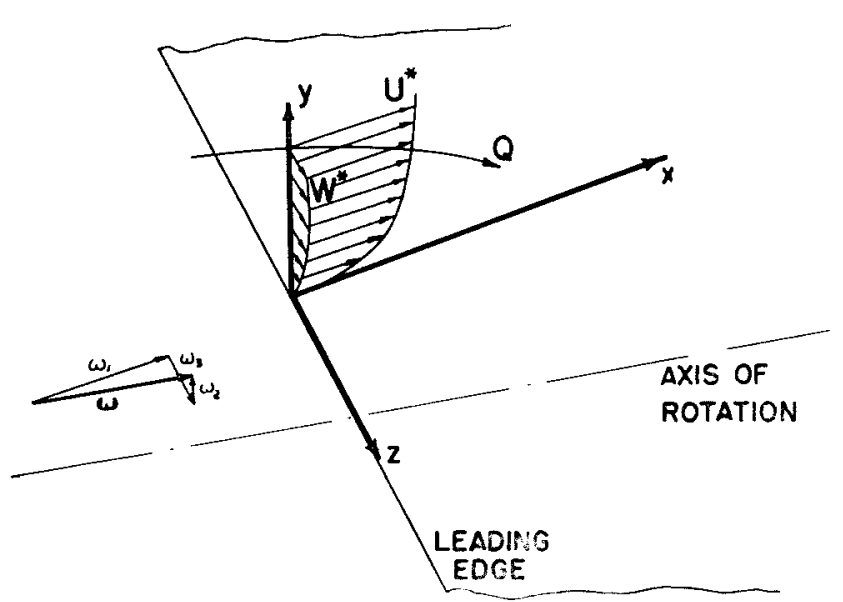

FIG. 1. Notation for flow over flat surface.

Since $U, W$, and $\omega_{2}$ will, in general, be different functions of $x$ and $z$, to satisfy Eqs. (9) for all values of these variables, it is necessary that the quantities in braces vanish separately. This is possible if all the coefficients of various powers of $x$ vanish independently, or in other words, all the expressions in brackets in Eqs. (9), when set equal to zero, become the differential equations which functions $f_{m}, f_{n}, f_{r}, g_{n}$, and $g_{r}$ must satisfy to make this true. Since the perturbation velocities (relative to the surface) are now given by

$$
\left.\begin{array}{rl}
\begin{array}{l}
u= \\
=
\end{array} \sum_{m} A_{m} x^{m} f_{m}{ }^{\prime}+\sum_{n} \frac{\partial B_{n}}{\partial z} x^{n+1} f_{n}{ }^{\prime}+ & 2 \sum_{r} \frac{\partial E_{r}}{\partial z} x^{r+2} f_{r}{ }^{\prime} \\
w= & \sum_{n} B_{n} x^{n} g_{n}{ }^{\prime}+2 \sum_{r} E_{r} x^{r+1} g_{r}{ }^{\prime} \\
v= & \frac{1}{2} \sqrt{\frac{\nu}{U^{0} x}} \times \\
\left\{\sum_{m} A_{m} x^{m}\left[(1+2 m) f_{m}-f_{m^{\prime}}^{\prime} \eta\right]+\right. \\
\sum_{n} \frac{\partial B_{n}}{\partial z} x^{n+1}\left[(2 n+3) f_{n}+2 g_{n}-f_{n}^{\prime} \eta\right]+ \\
\left.2 \sum_{r} \frac{\partial E_{r}}{\partial z} x^{r+2}\left[(2 r+5) f_{r}+2 g_{r}-f_{r}^{\prime} \eta\right]\right\}
\end{array}\right\}
$$

the appropriate boundary conditions are:

$$
\left.\begin{array}{r}
f_{m}(0)=f_{m}{ }^{\prime}(0)=f_{n}(0)=f_{n}{ }^{\prime}(0)=f_{r}(0)= \\
f_{r}^{\prime}(0)=g_{n}(0)=g_{n}{ }^{\prime}(0)=g_{r}(0)=g_{r}{ }^{\prime}(0)=0 \\
m^{\prime}(\infty)=g_{n}{ }^{\prime}(\infty)=1 ; \quad f_{n}^{\prime}(\infty)=f_{r}{ }^{\prime}(\infty)= \\
g_{r}{ }^{\prime}(\infty)=0
\end{array}\right\}
$$

As may be seen from Eqs. (9), the functions $g$ are completely independent of the functions $f$, which means that, to the first order of approximation, the cross-wise flow $w$ is completely independent of the principal flow perturbation $u$ and is affected only by the gradients of the external flow $W$ and the component of the angular velocity normal to the surface $\omega_{2}$. When the cross-wise velocity $w$ is not a function of the $z$ coordinate, the equations for the principal flow perturbation and the cross-wise flow perturbation are completely uncoupled. This means, that according to first-order theory, the changes in the principal flow velocity profile, and in particular the tendency toward the separation, are completely independent of the existence of cross-flow and will occur as if the flow were quasi-two-dimensional. This result is similar to the one obtained by Sears ${ }^{6}$ and applies even when the $u$ velocity varies in the $z$ direction. The cross-wise flow $w$ brings about changes in the principal velocity distribution in the form of functions $f_{n}{ }^{\prime}$ and $f_{\tau}{ }^{\prime}$, but only if that cross-wise flow is a function of the cross-wise coordinate $z$.

Two types of body forces were considered originally in Eq. (1): the centripetal force, and the Coriolis force. Of these, the centripetal force was assumed independent of the $y$ coordinate and consequently has been completely canceled out of the equations. The Coriolis force, which did depend on the $y$ coordinate, remained in the equations, and its effect demonstrates itself through functions $g_{r}{ }^{\prime}$ and $f_{r}{ }^{\prime}$. In internal aerodynamics, particularily in turbomachinery, it is often convenient to represent the external flow field as caused by some imaginary force field. It should be clear, from the above discussion, that such representation will have no effect on the boundarylayer flow except that caused by external flow velocities, provided the force field is not a function of the $y$ coordinate.

Solutions of Eqs. (9) for the various functions $f^{\prime}$ and $g^{\prime}$ were obtained by the relaxation method and are shown in Figs. 2(a-d). The inspection of these figures and Eqs. (9) reveals that the first-order solution does not affect the total boundary-layer thickness, which remains the same as that determined by the basic flow solution. This, of course, is not true for the momentum and displacement thicknesses, which will change, becautse of changes in the velocity profiles. We note also, that the maximum values of the functions $f_{n}{ }^{\prime}, f_{r}{ }^{\prime}$, and $g_{r}{ }^{\prime}$ are considerably lower than those of functions $f_{m}{ }^{\prime}$ and $g_{n}{ }^{\prime}$. This indicates that the effect of the Coriolis acceleration and of the variation of the $w$ velocity with $z$ coordinate is small when compared with the changes in velocity distribution brought about by the gradients of the external flow in the $x$ direction.

\section{Effect of Compressibility}

To estimate the effect of compressibility on threedimensional boundary-layer flow, one considers all quantities relative to some standard quantities, the viscosity as varying linearly with temperature, no heat transfer at the surface, and Prandtl Number equal to unity. Using Howarth's transformation, 


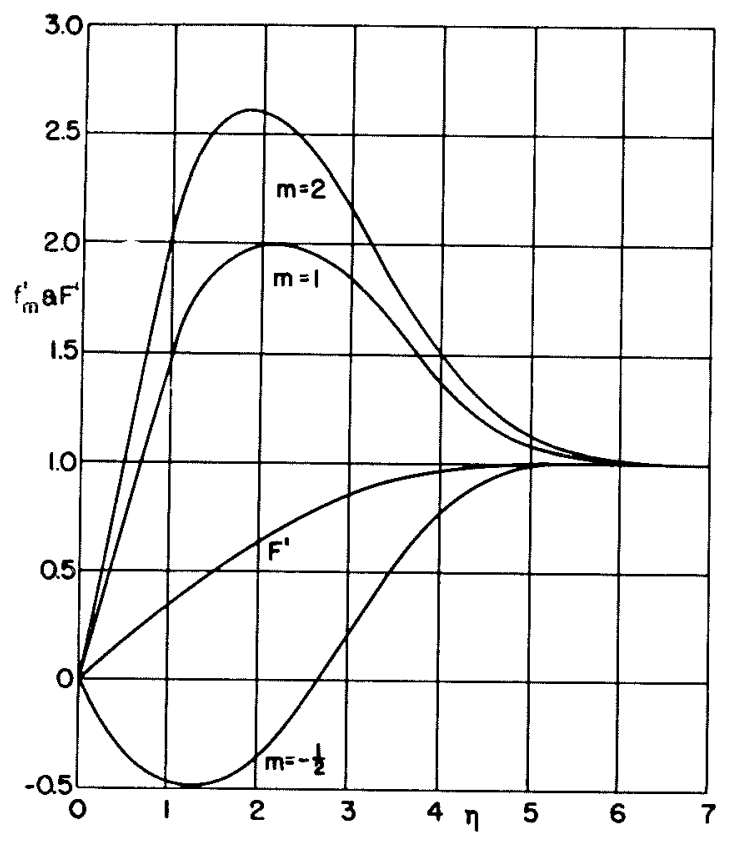

FIGURE 2. - PERTURBATION VELOCITY PROFILES

a) EFFECT OF PRESSURE GRAOIENT IN PRINCIPAL DIRECTION

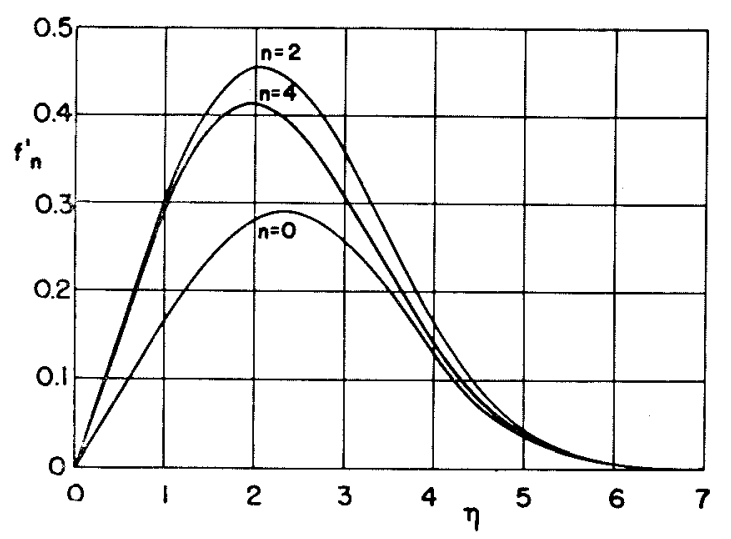

FIGURE 2. - CONT'D.

c) EFFECT OF CROSSWISE VARIATION OF THE CROSSWISE FLOW ON PRINCIPAL FLOW

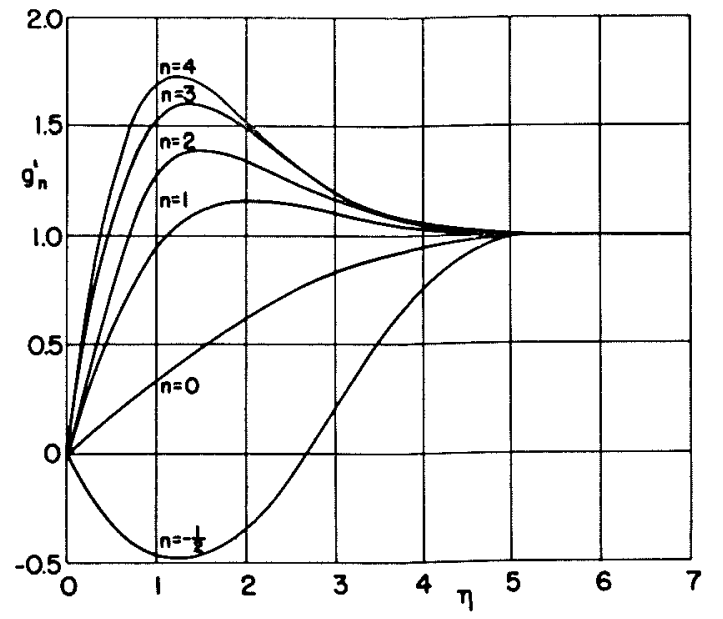

FIGURE 2.- CONT'D.

b) EFFECT OF PRESSURE GRADIENT IN CROSSWISE DIRECTION

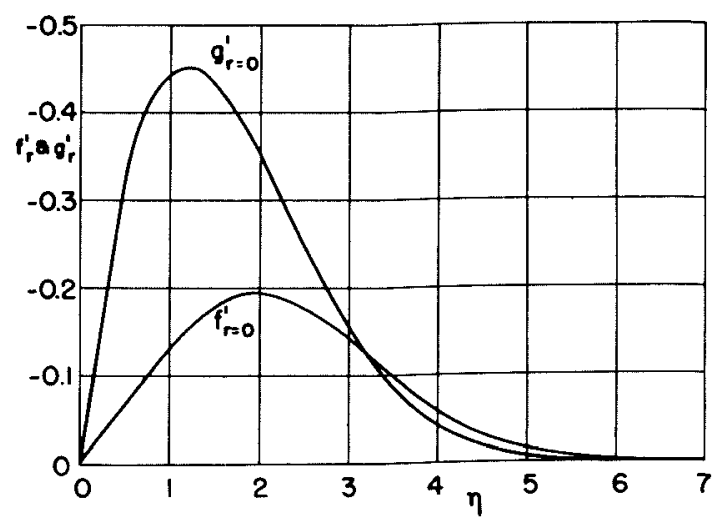

FIGURE 2. - CONT'D.

d) EFFECT OF SURFACE ROTATION $(r=0)$ 


$$
\left.\begin{array}{r}
Y \equiv\left(\frac{p}{p^{0}}\right)^{-1 / 2} \int_{0}^{y} \frac{\rho}{\rho^{0}} d y ; \quad X \equiv x ; \quad Z \equiv z \\
\psi \equiv\left(\frac{p}{p^{0}}\right)^{1 / 2} \bar{\psi} ; \quad \varphi \equiv\left(\frac{p}{p^{0}}\right)^{1 / 2} \bar{\varphi} ; \quad \bar{\eta} \equiv \\
Y \sqrt{\frac{U^{0}}{X}}
\end{array}\right\}
$$

$$
\begin{aligned}
\bar{\psi} & =\sqrt{U^{0} X}\left[\bar{F}+\frac{1}{U^{0}} \sum_{m} A_{m} X^{m} \bar{f}_{m}+\right. \\
\bar{\varphi} & =\sqrt{\frac{X}{U^{0}}}\left[\sum_{n} B_{n} X^{n} \bar{g}_{n}\right]
\end{aligned}
$$

yield again the Blasius equation as the zeroth-order equation. The first-order equations now are

The functions $\bar{\psi}$ and $\bar{\varphi}$ now written (for a nonrotating surface) as

$$
\begin{aligned}
& \left\{\sum _ { m } A _ { m } X ^ { m } \left\{\bar{f}_{m}^{\prime \prime \prime}+\frac{\bar{F}}{2} \bar{f}_{m}^{\prime \prime}-m \bar{F}^{\prime} \bar{f}_{m}^{\prime}+\left(\frac{1}{2}+m\right) \bar{F}^{\prime \prime} \bar{f}_{m}+m \times\right.\right. \\
& \left.\left.\left[1+\frac{\gamma-1}{2} M^{2}\left(1-\bar{F}^{\prime 2}\right)-\frac{\gamma}{2} M^{2} \bar{F} \bar{F}^{\prime \prime}\right]\right\}\right\}+ \\
& \left\{\sum_{n} \frac{\partial B_{n}}{\partial Z} X^{n+1}\left[\bar{f}_{n}^{\prime \prime \prime}+\frac{\bar{F}}{2} \bar{f}_{n}^{\prime \prime}-(n+1) \bar{F}^{\prime} \bar{f}_{n}{ }^{\prime}+\left(\frac{3}{2}+n\right) \bar{F}^{\prime \prime} \bar{f}_{n}+\bar{F}^{\prime \prime} \bar{g}_{n}\right]\right\}=0 \\
& \sum_{n} B_{n} X^{n}\left\{\bar{g}_{n}{ }^{\prime \prime \prime}+\frac{\bar{F}}{2} \bar{g}_{n}{ }^{\prime \prime}-n \bar{F}^{\prime} \bar{g}_{n}{ }^{\prime}+n\left[1+\frac{\gamma-1}{2} M^{2}\left(1-\bar{F}^{\prime 2}\right)\right]=0\right.
\end{aligned}
$$

Defining a new auxiliary function satisfying

$$
\begin{aligned}
\bar{f}_{m, c}{ }^{\prime \prime \prime}+\frac{\bar{F}}{2} \bar{f}_{m, c}^{\prime \prime}-m \bar{F}^{\prime} \bar{f}_{m, c}{ }^{\prime}+ \\
\left(\frac{1}{2}+m\right) \bar{F}^{\prime \prime} \bar{f}_{m, c}+\bar{F} \bar{F}^{\prime \prime}=0
\end{aligned}
$$

with boundary conditions

$$
\bar{f}_{m, c}{ }^{\prime}(0)=\bar{f}_{m, c}(0)=\bar{f}_{m, c}{ }^{\prime}(\infty)=0
$$

it is easy to show that the solution of the compressible equations is

$$
\begin{gathered}
\bar{f}_{m}=f_{m}(\bar{\eta})+\frac{\gamma-1}{2} M^{2}\left[f_{m}(\bar{\eta})-F(\bar{\eta})\right]- \\
\frac{M^{2}}{2}\left[m(2 \gamma-1)+\frac{\gamma-1}{2}\right] \bar{f}_{m, c} \\
\bar{f}_{n}=f_{n}(\bar{\eta})+\frac{\gamma-1}{2} M^{2}\left[f_{n}(\bar{\eta})-\bar{f}_{m, c}\right] \\
\quad(\text { with } m=n+1) \\
\bar{g}_{n}=g_{n}(\bar{\eta})+\frac{\gamma-1}{2} M^{2}\left[g_{n}(\bar{\eta})-F(\bar{\eta})\right]
\end{gathered}
$$

The Mach Number is thus removed from the differential equations and the solutions for the cross-flow of compressible fluid are constructed from those of incompressible fluid [note also that: $\bar{f}_{m=1, c^{\prime}}=f_{n=0^{\prime}}(\bar{\eta})$ ]. From these solutions it is apparent that compressibility of the fluid causes not only the characteristic "stretching" of the $y$ coordinate but also accentuates the crossflow.

\section{Examples}

In order to illustrate how these results are applied to actual cases of three-dimensional boundary-layer flow, two examples were computed. The first is taken from external aerodynamics and concerns a thin cylindrical shell of circular cross section, flying at a small angle of yaw along a circular path, and simultaneously spinning about its own axis of revolution (Fig. 3). The problem is to compute the boundary-layer flow on such a body. As has been shown by Howarth, ${ }^{1}$ all the results derived for a flat plate apply to the circular cylinder as well. For small angle of yaw, the external flow velocities are given by

$$
U^{0}=\Omega_{1} R ; \quad U=0 ; \quad W=2 \alpha \Omega_{1} R \sin \theta-\Omega_{2} a
$$

The angular velocity $\Omega_{2}$ has no component in the direction normal to the surface of the cylinder, so that
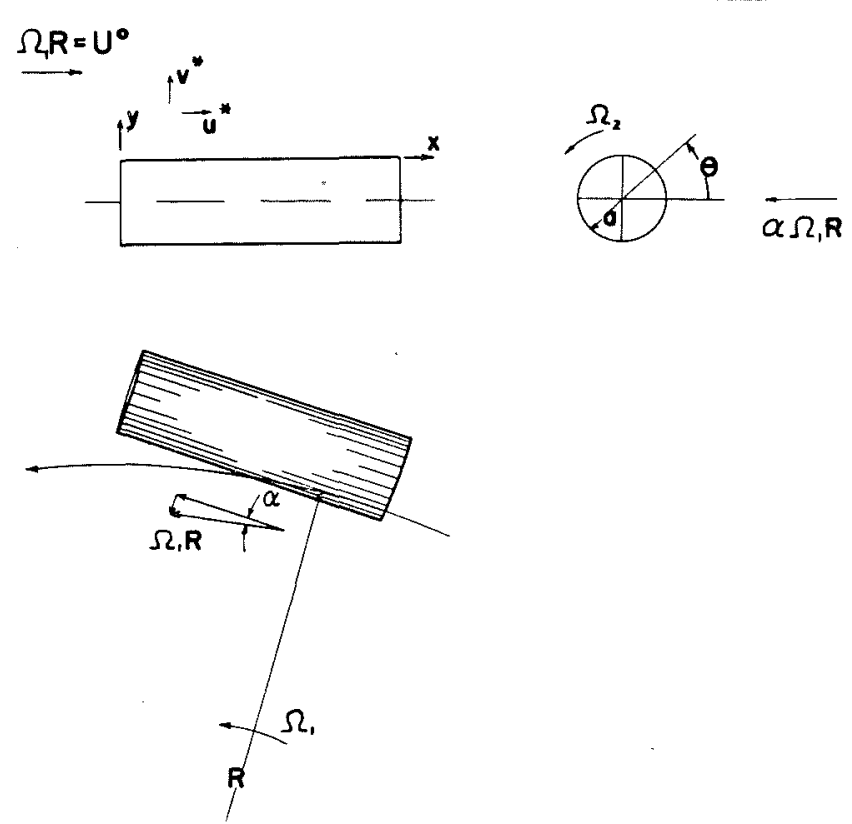

FIG. 3. Notation for flow over cylinder. 


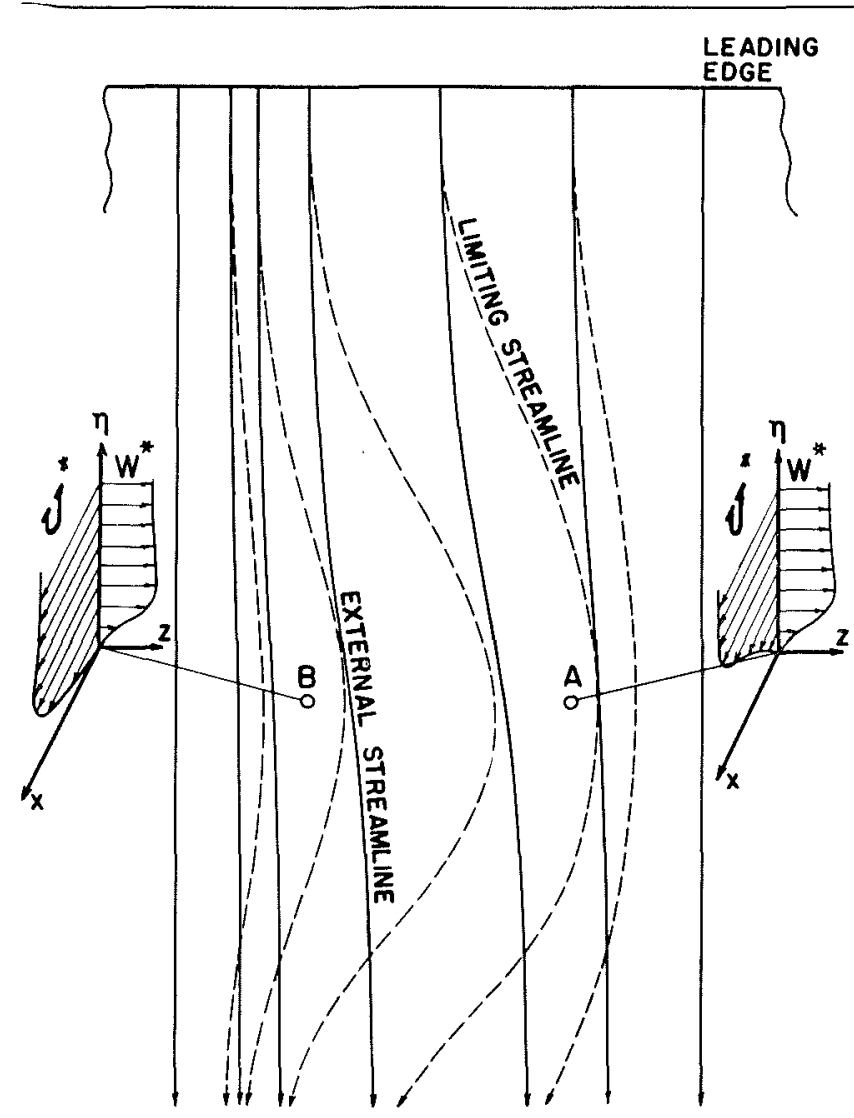

FIG. 4. Flow in simulated $s=$ shaped duct.

$\omega_{2}=\Omega_{1} \sin \theta . \quad$ Remembering that $z=a \theta$ and that for this case $n=r=0$, since $W$ and $\omega_{2}$ are independent of $x$, we write immediately, from Eqs. (10), the expressions for the $u^{*}$ and $w^{*}$ velocities in the boundary layer as

$$
\begin{aligned}
u^{*}=\Omega_{1} R F^{\prime}+2 \alpha \Omega_{1} R \frac{x}{a} \cos \theta f_{n=0^{\prime}} & + \\
& 2 x \Omega_{1} \frac{x}{a} \cos \theta f_{r=0}
\end{aligned}
$$

$w^{*}=\left(2 \alpha \Omega_{1} R \sin \theta-\Omega_{2} a\right) g_{n=0^{\prime}}+2 x \Omega_{1} \sin \theta g_{r=0}{ }^{\prime}$

The second example concerns the boundary-layer flow over a flat surface caused by a continuous force field simulating an s-shaped duct. Such flow is of interest, aside from the applications to internal ducting design, because it bears some resemblance to the flow over a casing of turbomachinery. In such machines, the successive rotor and stator stages serve to deflect the flow from the direction of the mean velocity, so that it oscillates about it in a periodic manner. In line with this analogy, the flow external to the boundary layer in this example is influenced by a body force in the crosswise direction, which is proportional to the square of the principal velocity, and the local curvature of the stream lines. This curvature, to simulate the double bend, is taken as

$c \approx\left(1-\cos 2 \pi \frac{z}{s}\right)\left[2\left(\frac{x}{L}\right)-6\left(\frac{x}{L}\right)^{2}+4\left(\frac{x}{L}\right)^{3}\right]$
In addition, the velocity in the principal direction is assumed to be a constant. After cross differentiation, to eliminate the pressure, the Euler's equations yield

$$
U^{0}\left(W_{x x}-U_{x z}\right)=\left(G_{3}\right)_{x}-\left(G_{1}\right)_{z}
$$

so that (since $U=G_{2}=0$ ) the external velocity is given by

$$
\begin{aligned}
U^{*}= & U^{0} ; \quad W^{*}=W=16 \tan \vartheta U^{0} \times \\
& \left(1-\cos 2 \pi \frac{z}{s}\right)\left[\left(\frac{x}{I}\right)^{2}-2\left(\frac{x}{L}\right)^{3}+\left(\frac{x}{L}\right)^{4}\right]
\end{aligned}
$$

The integration constants determining the initial value of the body force and the inclination of the simulated duct have been arbitrarily set to zero. The picture of the stream lines (with the average deflection $\vartheta$ equal to $5^{\circ}$, and the width of duct $s$, equal to half its length $I$ ), is shown in Fig. 4.

Since the body force varies only in $x$ and $z$ direction, the boundary-layer velocities can be written, using Eqs. (10), as

$$
\begin{array}{r}
u^{*}=U^{0}\left\{F^{\prime}+32 \pi \tan \vartheta \sin \left(2 \pi \frac{z}{s}\right)\left(\frac{L}{s}\right)\left(\frac{x}{L}\right)^{3} \times\right. \\
\left.\left[f_{n=2^{\prime}}-2\left(\frac{x}{L}\right) f_{n=3^{\prime}}+\left(\frac{x}{L}\right)^{2} f_{n=4^{\prime}}\right]\right\} \\
w^{*}=16 \tan \vartheta U^{\circ}\left(1-\cos 2 \pi \frac{z}{s}\right)\left(\frac{x}{L}\right)^{2} \times \\
{\left[g_{n=2^{\prime}}-2\left(\frac{x}{L}\right) g_{n=3^{\prime}}+\left(\frac{x}{L}\right)^{2} g_{n=4^{\prime}}\right]}
\end{array}
$$

From the knowledge of the velocity distributions, one can compute the shape of the "limiting stream lines" at the wall $(y=0)$, and these are also shown in Fig. 4 . It can be seen in this figure, that the boundary layer flows toward the "wall of the duct" nearest to the center of curvature. If the duct is to simulate two rows of blades, this means boundary layer flows toward the suction surface of the first row of the blades. By studying the velocity distributions at points $A$ and $B$ (in Fig.

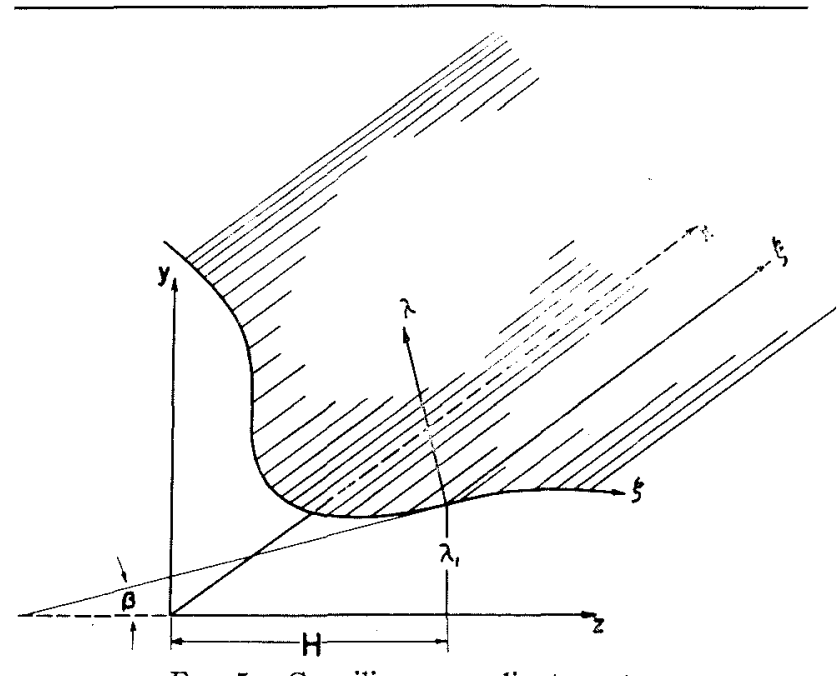

FIG. 5. Curvilinear coordinate system. 
4 the scale of $W^{*}$ is enlarged out of proportion), we note that the shift of the boundary-layer flow implies also a redistribution of the momentum of the principal flow, since the principal flow at point $A$ slows down and tends toward separation, while that at point $\mathrm{B}$ accelerates. In fact, since in the vicinity of point $A$ the crosswise flow also changes direction, somewhere near that point may occur a true separation of three-dimensional boundary layer-that is, all of the flow will leave the surface. The change of direction of the limiting stream lines occurs further downstream than the inflection point of the external flow. This means, that in the second row of blades the boundary layer will first continue toward the pressure surface (where it may separate) and only afterwards, deflect again toward the suction surface.

\section{(III) Boundary-Layer Flow over Sharply Curved SURFACE}

\section{Perturbed Equations in Curvilinear System}

In the last example, the "sidewalls" of the duct were straight, starting from the leading edge, and therefore, no cross-flow occurred there. In actuality, however, the sides would be curved, and, since the experimental evidence points strongly to the corners between these walls and the bottom of the duct as regions of high total pressure loss, the flow in such corners should also be studied. To simplify matters, one considers the corners as filleted, so that the boundary layer flows over a sharply (but continuously) curved surface.

For the purpose of this analysis it is convenient to use an orthogonal curvilinear coordinate system shown in Fig. 5. The transformation from the Cartesian system $x, y, z$ to to the curvilinear system $\xi, \lambda, \xi$ is given by

$$
\begin{array}{r}
x \equiv \xi ; y \equiv \lambda_{1}+\lambda \cos \beta ; \lambda_{1} \equiv H-\int_{0}^{\zeta} \sin \beta d \zeta ; \\
z \equiv \int_{0}^{\zeta} \cos \beta d \zeta+\lambda \sin \beta
\end{array}
$$

Restricting the analysis to curvature of the $\zeta$ axis $c=d \beta / d \zeta$, being a function of $\zeta$ coordinate only, the length stretching factors used in the transformation of the equations of motion become

$$
h_{1}=1 ; \quad h_{2}=1 ; \quad h_{3}=1+c \lambda
$$

It should be pointed out that in this coordinate system the $\zeta$ axis plays the role of a reference line and will be used to describe the shape of the wall over which the fluid is flowing. Coordinate $\lambda$ will then measure the distance perpendicular to this wall, while the $\xi$ axis is pointed in the direction of the principal flow, so that $\xi=$ $\lambda=0$ represent the leading edge of the configuration. Using the standard transformation formulas (velocities $u^{*}, v^{*}, w^{*}$ are taken here in the $\xi, \lambda, \zeta$ directions, respectively) and applying the boundary-layer approximations-that is, considering $v^{*}$ and $\lambda$ of $0(\delta), c$ of $0(1 / \delta)$ or larger, and neglecting all terms of $0(\delta)$ - one obtains the boundary-layer momentum equations,

$$
\begin{gathered}
u^{*} u_{\xi}^{*}+v^{*} u_{\lambda}^{*}+\frac{1}{1+c \lambda} w^{*} u_{\zeta}^{*}= \\
-\frac{1}{\rho} p_{\xi}+\nu\left(u_{\lambda \lambda}^{*}+\frac{c}{1+c \lambda} u_{\lambda}^{*}\right) \\
{[c /(1+c \lambda)]\left(w^{*}\right)^{2}=-(1 / \rho) p_{\lambda}} \\
u^{*} w_{\xi}+v^{*} w_{\lambda}^{*}+\frac{1}{1+c \lambda} w^{*} w_{\zeta}^{*}+ \\
\frac{c}{1+c \lambda} v^{*} w^{*}=-\frac{1}{\rho} \frac{1}{1+c \lambda} p_{\zeta}+\nu \times \\
{\left[w_{\lambda \lambda}^{*}+\frac{c}{1+c \lambda} w_{\lambda}^{*}-\left(\frac{c}{1+c \lambda}\right)^{2} w^{*}\right]}
\end{gathered}
$$

and the equation of continuity,

$$
u_{\xi}^{*}+v_{\lambda}^{*}+\frac{1}{1+c \lambda} w_{\lambda}^{*}+\frac{c}{1+c \lambda} v^{*}=0
$$

One now considers the flow as only slightly perturbed from the $\xi$ direction, so that the zeroth-order terms yield again the basic flow equations,

$$
\left.\begin{array}{l}
u^{0} u_{\xi}^{0}+v^{0} u_{\lambda}^{0}=\nu\left\{u_{\lambda \lambda}^{0}+[c /(1+c \lambda)] u_{\lambda}^{0}\right\} \\
u_{\xi}^{0}+v_{\lambda}^{0}+[c /(1+c \lambda)] v^{0}=0
\end{array}\right\}
$$

and the first-order terms give the perturbation equations,

$$
\begin{aligned}
u^{0} u_{\xi}+u u_{\xi}^{0}+v^{0} u_{\lambda}+v u_{\lambda}^{0}+\frac{1}{1+c \lambda} w u_{\xi}^{0}= \\
-\frac{1}{\rho} p_{\xi}+\nu\left(u_{\lambda \lambda}+\frac{c}{1+c \lambda} u_{\lambda}\right) \\
u^{0} w_{\xi}+v^{0} w_{\lambda}+\frac{c}{1+c \lambda} v^{0} w= \\
-\frac{1}{\rho} \frac{1}{1+c \lambda} p_{\zeta}+\nu\left[w_{\lambda \lambda}+\frac{c}{1+c \lambda} w_{\lambda}-\right. \\
\left.\left(\frac{c}{1+c \lambda}\right)^{2} w\right] \\
p_{\lambda}=0\left(w^{2}\right) \quad v_{\lambda}+\frac{1}{1+c \lambda} w_{\zeta}+\frac{c}{1+c \lambda} v=0
\end{aligned}
$$

\section{Solution of Basic Flow Equations}

The basic flow equations may be solved by defining a stream function $\psi^{0}(\xi, \lambda, \zeta)$, and an additional parameter $\kappa=c\left(\nu \xi / U^{0}\right)^{1 / 2}$ related to the local curvature of the wall. The stream function is first expanded,

$$
\psi^{0}=\sqrt{U^{0} \nu \xi}\left[F(\eta)+\kappa d(\eta)+\kappa^{2} d_{1}(\eta)+\ldots\right]
$$

and introduced in this form into the equations of basic flow. Again, since the $d$ 's must be independent of $\zeta$, the coefficients of the various powers of $\kappa$ are set separately to zero. The maximum permissible value of $\kappa$ will be small [of $0\left(1 / \eta_{\delta}\right)$ ], otherwise the curvilinear system would lead to double values, and, consequently, 


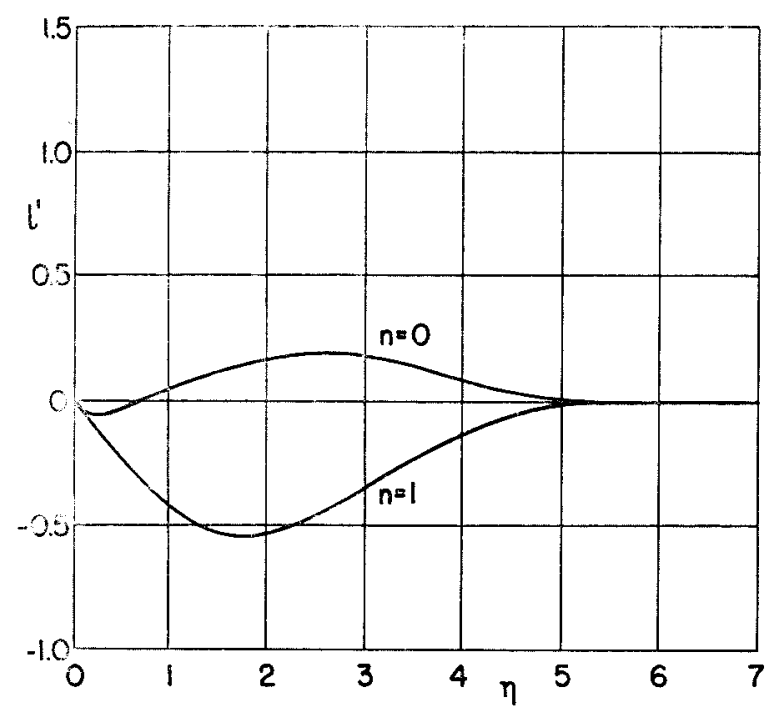

FIGURE 6. - VELOCITY PERTURBATION DUE TO WALL CURVATURE

a) IN THE PRINCIPAL DIRECTICN

it is sufficient to consider only the first two terms in this expansion. One thus obtains the Blasius equation for $F(\eta)$ and an equation for $d$ as follows:

$$
\begin{gathered}
2 d^{\prime \prime \prime}+2 d d^{\prime \prime}-\left(d^{\prime}\right)^{2}+(\eta F-2) F^{\prime \prime}+ \\
F^{\prime}\left(F^{\prime} \eta-F\right)=0
\end{gathered}
$$

Since the basic flow velocities, neglecting higher powers of $\kappa$, are:

$$
\left.\begin{array}{rl}
u^{0} \doteq[1 /(1+\kappa \eta)] U^{0}\left(F^{\prime}+\kappa d^{\prime}\right) & \\
v^{0} \doteq-\frac{1}{2} \frac{1}{1+\kappa \eta} \sqrt{\frac{U^{0} \nu}{\xi}}\left[\left(F-F^{\prime} \eta\right)+\right. \\
\left.\kappa\left(2 d-d^{\prime} \eta\right)\right]
\end{array}\right\}
$$

$d$ must satisfy $d(0)=d^{\prime}(0)=0$ and $d^{\prime}(\infty)=\eta$. A numerical solution for $d^{\prime}$ (reference 15) shows that $d^{\prime} \approx \eta$ everywhere, and therefore a fair approximation for the $u^{0}$ velocity is

$$
u^{0} \doteq U^{0}\left[\left(F^{\prime}+\kappa \eta\right) /(1+\kappa \eta)\right]
$$

This approximation has been used subsequently. Since, for small values of $\eta, F^{\prime}$ may be approximated by $F^{\prime}=0.332 \eta$, expression (25) shows that, for small values of $\eta, u^{0} \doteq(0.332+\kappa) \eta+0\left(\eta^{2}\right)$. In other words, the slope of the velocity distribution near the surface depends to a great extent on the local curvature of this surface. On concave surfaces, $\kappa$ will be negative, and thus the velocity profile will be nearer separation (will have a smaller slope), and on convex surfaces the basic flow velocity profile improves.

\section{Solution of the Perturbed Equations}

To solve the first-order equations, one proceeds in exactly the same manner ${ }^{15}$ as for basic flow. To sim-

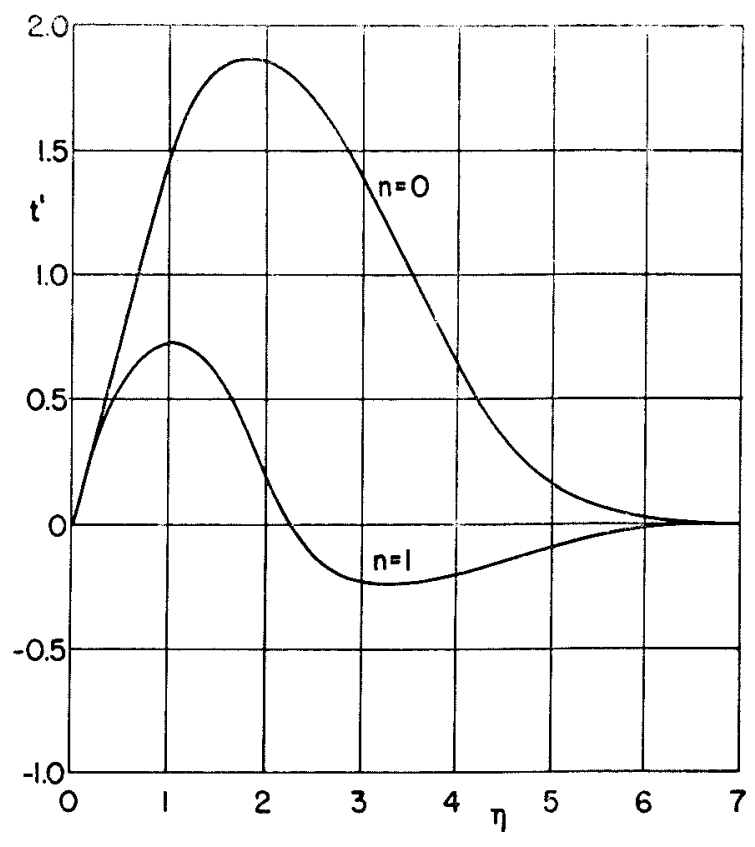

FIGURE 6.-CONT'D.

b) IN THE CROSS:VISE CIRECTION

plify matters but still retain a significant model, it is sufficient to consider the external flow to be of special type: $U=0, W=D \xi^{n}$. The appropriate expressions for $\psi$ and $\varphi$ may now be written immediately, as a result of the flat surface investigation,

$$
\left.\begin{array}{c}
\psi(\xi, \eta, \zeta)=\xi \frac{\partial \kappa}{\partial \zeta} \frac{W}{U^{0}} \sqrt{\nu \xi U^{0}} \times \\
{\left[l_{n}(\eta)+\kappa l_{1, n}(\eta)+\ldots\right]} \\
\varphi(\xi, \eta, \zeta)=\frac{W}{U^{0}} \sqrt{\nu \xi U^{0}}\left[g_{n}(\eta)+\right. \\
\left.\kappa t_{n}(\eta)+\kappa^{2} t_{1, n}(\eta)+\ldots\right]
\end{array}\right\}
$$

It is clear that the equation for $g_{n}$ must be the same as for the flat surface, and the equations for $l_{n}$ and $t_{n}$ become

$$
\left.\begin{array}{r}
l_{n}^{\prime \prime \prime}+\frac{F}{2} l_{n}^{\prime \prime}-\left(\frac{3}{2}+n\right) F^{\prime} l_{n}^{\prime}+ \\
(2+n) F^{\prime \prime} l_{n}+F^{\prime \prime} t_{n}-\left(d^{\prime}-F^{\prime} \eta\right) g_{n}^{\prime}=0 \\
t_{n}^{\prime \prime \prime}+\frac{F}{2} t_{n}^{\prime \prime}-\left(\frac{1}{2}+n\right) F^{\prime} t_{n}^{\prime}-\eta g_{n}{ }^{\prime \prime \prime}+ \\
\left(d+\frac{F}{2} \eta+1\right) g_{n}^{\prime \prime}-\left[n\left(F^{\prime} \eta+d^{\prime}\right)+\right. \\
\left.\frac{1}{2}\left(F^{\prime} \eta-F\right)\right] g_{n}^{\prime}+2 n \eta+ \\
\frac{1}{2} \lim _{\eta \rightarrow \infty}\left(F^{\prime} \eta-F\right)=0
\end{array}\right\}
$$

Since the perturbation velocity components are 


$$
\begin{gathered}
u=W \frac{d(\log c)}{d \zeta} \frac{c \xi^{2}}{1+c \lambda} \frac{1}{\sqrt{R e}} l_{n}{ }^{\prime} \\
w=W\left[g_{n}{ }^{\prime}+c \xi \frac{1}{\sqrt{\operatorname{Re}}} t_{n}{ }^{\prime}\right]
\end{gathered}
$$

the appropriate boundary conditions are:

$$
l_{n}(0)=l_{n}{ }^{\prime}(0)=l_{n}{ }^{\prime}(\infty)=t_{n}(0)=t_{n}{ }^{\prime}(0)={ }_{t_{n}{ }^{\prime}(\infty)=0}
$$

Equations (27) were solved numerically for $n=0$ and $n=1$. These solutions are shown in Figs. $6 \mathrm{a}$ and $6 \mathrm{~b}$.

\section{Example}

To illustrate the manner in which the derived results are applied to an actual boundary-layer flow' over a sharply curved wall, such flow has been computed for $U=0, W=D \xi^{n}$, with $n=0$ and $n=1$. The wall was assumed to have a hyperbolic shape. Assuming $\eta_{\delta}=8.0$, the maximum permissible value of $\kappa$ has been set at 0.125 , so that at any given Reynolds Number $U^{0} / \nu c_{\text {max. }}$, there exists a maximum value of $c \xi$ :

$$
c_{\max .} \xi_{\max .}=(0.125)^{2} U^{0} / \nu c_{\max }
$$

for which these considerations hold. In this example

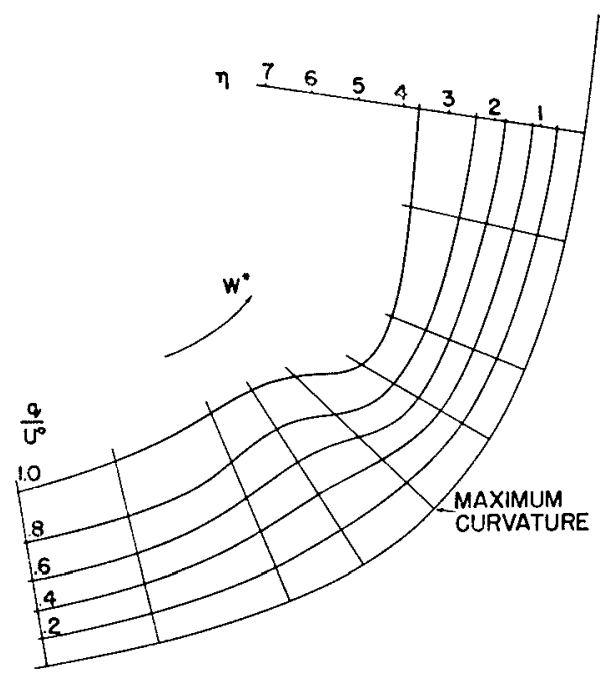

FIGURE 7- FLOW OVER A HYPERBOLICALLY SHAPED WALL

a) EXTERNAL FLOW: $U^{*}=U^{\circ} ; W^{*}=0$ CONSTANT VELOCITY PROFILES

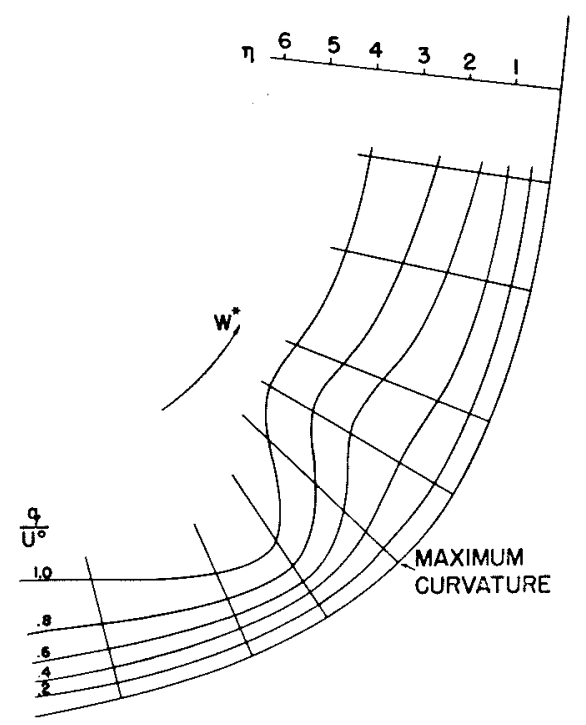

FIGURE 7. - CONT'D.

c) EXTERNAL FLOW: $U^{*}=U^{\circ} ; W^{*}=D$ * CONSTANT VELOCITY PROFILES

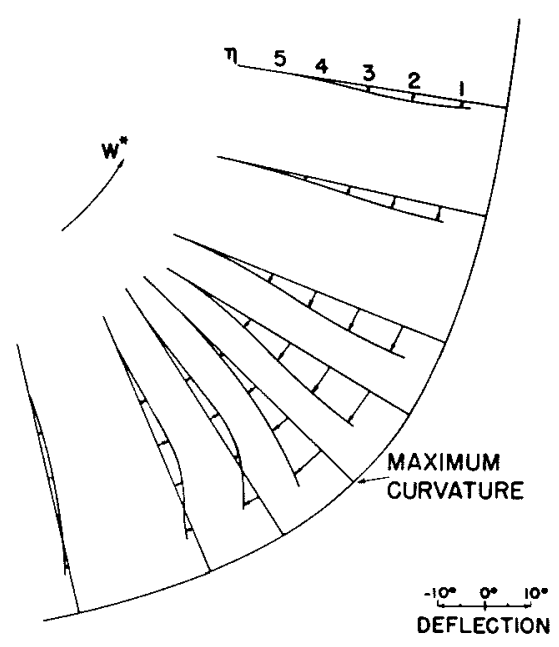

FIGURE 7.- CONT'D.

b) EXTERNAL FLOW: $U^{*}=U^{\circ} ; W^{*}=D$ DEFLEGTION FROM EXTERNAL FLOW DIRECTION

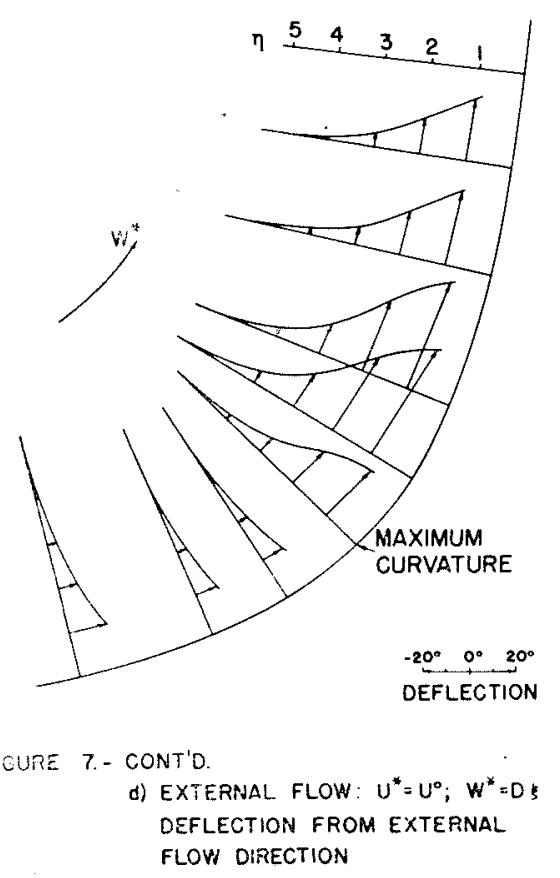


$U^{0} / \nu c_{\max .}=634$, so that $\xi_{\max .}=10 / c_{\max }$. The results of the computations are shown in Figs. $7(\mathrm{a}-\mathrm{d})$, where are plotted the velocity ratios, and the deflection from the external flow direction. It should be noted that at the computed position $\xi=\xi_{\text {max. }}$, the cross-wise component of the external flow $W^{*}$ is the same in both cases, so that the differences can be traced directly to the cross-wise pressure gradient. Physically, such an external flow may be thought of as a vortex of constant strength for $n=0$, and a vortex of an increasing strength for $n=1.0$. Examination of Figs. $7 \mathrm{a}$ and $7 \mathrm{~b}$ shows that, for $n=0$, an accumulation of low momentum air occurs on the "upstream" wall of the corner. When $n=1.0$, however, Figs. $7 \mathrm{c}$ and $7 \mathrm{~d}$ reveal that now the large accumulation of the low momentum air occurs on the "downstream" wall, with corresponding large deflections in that region. We thus see that, when there is no pressure gradient in the external flow $(n=0)$, the wall curvature causes a weak secondary flow as shown in Fig. 7b. When, on the other hand, the external flow has a cross-wise pressure gradient $(n=1)$, the secondary flow due to that pressure gradient apparently (Fig. 7d) is in such a direction as to hinder the principal flow on the "downstream" wall, so that almost all of the flow "climbs" the wall in that region.

\section{(IV) Concluding Remarks}

The analysis of three-dimensional, steady laminar boundary layer with small cross-flow has indicated that many important problems in this class can be solved with relatively little effort, thus affording a greater understanding of real fluid flow. Although the accurate determination of the separation point is certainly beyond the scope of small perturbation analysis (and also beyond the standard boundary-layer theory), the factors that affect the separation of three-dimensional boundary layers are clearly pointed out. Among these factors, the cross-wise variation of flow is indicated as important in affecting the separation. In particular, the examples show the strong tendency toward the separation as a result of cross-wise variation of cross-flow, both on a cylindrical shell and in a simulated s-shaped duct. Similarily, the sharp, laterally varying, concave curvature of the surface tends to separate even the basic flow profile.

\section{REFERENCES}

${ }^{1}$ Howarth, L., The Boundary Layer in Three-Dimensional Flow-Part I. Derivation of the Equations for Flow Along a General Curved Surface, Philosophical Mag., Vol. XLII, pp. 239-243, March, 1951

2 Hayes, Wallace D., The Three-Dimensional Boundary Layer, NAVORD Rep. 1313, NOTS 384, U.S. Naval Ordnance Test Station (Inyokern), May 9, 1951.

${ }^{3}$ Moore, Franklin K., Three-Dimensional Compressible Laminar Boundary Layer Flow, NACA TN No. 2279, 1951.

${ }^{4}$ Timman, R., A Calculation Method for Three-Dimensional Laminar Boundary Layers, National Luchtvaartlab. Amsterdam, Rep. F. 66, 1951.

${ }^{5}$ Mager, A., Generalization of Boundary-Layer MomentumIntegral Equations to Three-Dimensional Flows Including Those of Rotating System, NACA TR 1067, 1952.

${ }^{6}$ Sears, W. R., The Boundary Layer of Yawed Cylinders, Journal of the Aeronautical Sciences, Vol. 16, No. 1, pp. 41-45, January, 1949.

${ }^{7}$ Bödewart, U. T., Die Drehströmung über festem Grunde, ZAMM, 20, 241, 1940 .

- Howarth, L., Note on the Boundary Layer on a Rotating Sphere, Philosophical Mag., Ser. 7, Vol. XLII, pp. 1308-1315, November, 1951.

- Loos, H. G., A Simple Laminar Boundary Layer with Secondary Flow, Daniel and Florence Guggenheim Jet Propulsion Center, California Institute of Technology, Technical Report No. 1.

${ }^{10}$ Carrier, G. F., The Boundary Layer in a Corner, Quart. of Appl. Math., Vol. IV, No. 4, pp. 367-370, January, 1947.

${ }^{11}$ Moore, F. K., Laminar Boundary Layer on a Circular Cone in Supersonic Flow at a Small Angle of Attack, NACA TN No. $2521,1951$.

${ }^{12}$ Moore, F. K., Three-Dimensional Boundary Layer Flow, Journal of the Aeronautical Sciences, Vol. 20, No. 8, pp. 525534, August, 1953.

${ }^{13}$ Fogarty, L. E., The Laminar Boundary Layer on a Rotating Blade, Journal of the Aeronautical Sciences, Vol. 18, No. 4, pp. 247-252, Apri1, 1951

${ }^{14}$ Schlichtling, H., Lecture Series-Boundary Layer TheoryPart I-Laminar Flows, NACA TM No. 1217, 1949.

${ }_{15}$ Mager, A., Laminar Boundary Layer Problems Associated with Flow Through Turbomachines, Doctoral Thesis, California Institute of Technology, 1953. 